Cabe destacar que la desigualdad de género que se observó no solo abarca al mundo académico, ya que se muestra en otros ámbitos laborales y aún impacta en el desempleo, que afecta mayormente a las mujeres, en especial a las más jóvenes. ${ }^{15}$

\section{CONCLUSIÓN}

La presencia de mujeres autoras en artículos publicados en revistas de América Latina se ha incrementado en forma sostenida en las últimas décadas. En este estudio, la proporción de mujeres autoras en tres revistas pediátricas latinoamericanas alcanzó el 59,9\%. La participación como primeras o últimas autoras fue significativamente menor en Archivos Argentinos de Pediatría.

\section{Agradecimiento}

Los autores agradecen a los presidentes de la Sociedad Argentina de Pediatría, Sociedade Brasileira de Pediatria y Sociedad Chilena de Pediatría, quienes gentilmente compartieron los datos sobre sus respectivas sociedades.

\section{REFERENCIAS}

1. FilardoG, da GracaB, Sass DM, etal. Trends and comparison of female first authorship in high impact medical journals: observational study (1994-2014). BMJ 2016;352:i847.

2. Argentina. Ministerio de Salud. Observatorio Nacional de Recursos Humanos en Salud. Los recursos humanos de salud en la Argentina. 2015. [Acceso: 23de diciembre de 2016]. Disponible en: http://www.msal.gob.ar/ observatorio/images / stories / documentos_fuerza_ trabajo/RHuS_ARG_2015.pdf.

3. Scheffer M, Cassenote A. A feminização da medicina no Brasil. Ver Bioet 2013;21(2):268-77.
4. Instituto Nacional de Estadística, Chile. Compendio Estadístico. 1.6 Estadísticas de Salud 2010. [Acceso: 3 de abril de 2017]. Disponible en: http://www.ine.cl/docs/defaultsource/publicaciones/2010/compendio_2010.rar?sforsn=5.

5. Universidad de Buenos Aires. Censo de estudiantes 2011. [Acceso: 16 de diciembre de 2016]. Disponible en: http:// www.uba.ar/institucional/censos/Estudiantes2011/ estudiantes2011.pdf.

6. Universidad de Buenos Aires. Censo de docentes 2011 [Acceso: 16 de diciembre de 2016].Disponible en:www. uba.ar/institucional/censos/Docentes2011/docentes2011final.pdf.

7. West JD, Jacquet J, King MM, et al. The Role of Gender in Scholarly Authorship. PLoSOne 2013;8(7):e66212.

8. FerreroF, OteroP.Participación dela mujeren publicaciones periódicas científicas: tendencias en la pediatría latinoamericana. Arch Argent Pediatr 2007;105(3):244-7.

9. UNESCO InstituteforStatistics. Mujeres en ciencia. Investigadoras por país. [Acceso: 4 de abril de 2017]. Disponible en:http:/ / www.uis.unesco.org/_LAYOUTS / UNESCO/ women-in-science/index.html?t=149124378263 7 \# overview!lang $=$ es\&region $=40520$.

10. Red deIndicadores de Ciencia y Tecnología Iberoamericana e Interamericana. Recursos humanos. Personal por género. [Acceso: 12 de diciembre de 2016]. Disponible en: http: / / db.ricyt.org/ query / AR,BO,CL,CO,CR,CU,EC,ES ,GT,HN,NI,PA,PR,PT,PY,SV,TT,UY,VE / 1990\%2C2014/ PERSOPFGENPER.

11. Franchi A, Kochen S, Maffía D, et al. Evolución de la situación delas Mujeres en el sector de Ciencia y Tecnología en Argentina (1998-2013). X Congreso Iberoamericano de Ciencia, Tecnología y Género. 28 a 30 de octubre de 2014. - Asunción, Paraguay; 2014.

12. Macaluso $B$, Larivière $V$, Sugimoto $T$, et al. Is Science Built on the Shoulders of Women? AStudy of Gender Differences in Contributorship. Acad Med 2016;91(8):1136-42.

13. Fridner A, Norell A, Åkesson G, et al. Possible reasons why female physicians publish fewer scientific articles than male physicians - a cross-sectional study. BMC Med Educ 2015;15:67.

14. DelBocaFK.Addressingsexand genderinequitiesinscientific research and publishing. Addiction 2016;111(8):1323-5.

15. Organización Internacional del Trabajo. Las mujeres en el trabajo. Tendencias 2016. Resumen ejecutivo. Ginebra, 2016. [Acceso: 4 deabril 2017]. Disponible en:http: / / www. unesco.org/library/PDF/wcms_457094.pdf

\title{
Estrategias para el cumplimiento del programa de formación en tres médicos residentes de Pediatría con alergia al látex
}

\section{Strategies for compliance with the internship program among three pediatric interns with latex allergy}

Dra. Stefanía Barbariol ${ }^{a}$ Dr. Alfredo Eymann ${ }^{b, c}$, Dr. Julián Llera ${ }^{c}$ y Dr. Claudio A. Parisi ${ }^{a}$

a. Sección de Alergia del Servicio de Clínica Pediátrica del Hospital Italiano de Buenos Aires, Buenos Aires, Argentina.

b. Departamento de Posgrado del Instituto Universitario del Hospital Italiano de Buenos Aires, Buenos Aires, Argentina.

c. Servicio de Clínica Pediátrica del Hospital Italiano de Buenos Aires, Buenos Aires, Argentina.

\author{
Correspondencia: \\ Dr. Claudio A. Parisi: claudio.parisi@hospitalitaliano.org.ar \\ Financiamiento: Ninguno.
}

Conflicto de intereses: Ninguno que declarar.

Recibido: 28-2-2017

Aceptado: 28-6-2017 


\section{RESUMEN}

Introducción. La alergia al látex es frecuente en ámbitos hospitalarios.

Objetivo. Describir la situación clínica de tres residentes de Pediatría con alergia al látex y las estrategias de prevención en las rotaciones de Terapia Intensiva Pediátrica y Neonatal. Pacientes, métodos y resultados. Las tres profesionales manifestaron exacerbación de síntomas durante la residencia. Se confirmó el diagnóstico con historia compatible e inmunoglobulinaE específica positiva.Se realizó una entrevista semiestructurada para describir sus percepciones en relación con las estrategias de prevención, se capacitó al personal y se incorporaron guantes de nitrilo para los procedimientos.

Las residentes realizaron las rotaciones sin presentar manifestaciones alérgicas. Refirieron como aspectos positivos sentirse cuidadas y mejor calidad de vida; como aspectos negativos, mala predisposición y resistencia al cambio en algunos compañeros.

Las estrategias diseñadas permitieron que las residentes pudieran continuar su programa de formación.

Palabras clave: alergia al látex, residencia médica, prevención, trabajadores de salud.

http: / / dx.doi.org/10.5546/ aap.2017.583

Texto completo en inglés:

http:/ / dx.doi.org/10.5546/ aap.2017.eng.583

Cómo citar: Barbariol S, Eymann A, Llera J, et al. Estrategias para el cumplimiento del programa de formación en tres médicos residentes de Pediatría con alergia al látex. Arch Argent Pediatr 2017;115(6):583-587.

\section{INTRODUCCIÓN}

La alergia al látex se describió por primera vez en Alemania, en 1927. ${ }^{1}$ A partir de la década del ochenta, los casos reportados aumentaron de modo considerable y esto se debió, principalmente, a tres factores: a) uso masivo de guantes de látex para evitar el contagio de enfermedades, b) disminución en la calidad de producción con aumento de su contenido proteico y c) sustitución del talco por almidón de maíz para prevenir la formación de granulomas.

El uso frecuente de guantes de látex en áreas cerradas, tales como los quirófanos, genera una atmósfera de alta densidad de partículas de almidón de maíz, que, por su alto peso molecular, es capaz de adherir en su superficie moléculas más pequeñas, como el látex, $\mathrm{y}$, de esa manera, vehiculizarlas. ${ }^{2}$

Las manifestaciones clínicas varían dependiendo de la ruta de exposición: pueden presentarse en forma de manifestaciones leves, como urticaria de contacto o rinoconjuntivitis, hasta reacciones graves, como anafilaxia. ${ }^{3}$

La residencia médica es un sistema de socialización profesional en el que, a través de una estrategia de inmersión, los jóvenes profesionales se apropian de los valores, normas, prácticas, conocimientos del grupo al que aspiran pertenecer y permite transformar a un médico recién recibido en un experto. ${ }^{4}$

Los programas incluyen el aprendizaje de prácticas y procedimientos que requieren, para su bioseguridad, el uso de material con látex. El riesgo de alergia se encuentra incrementado en escenarios con una elevada tasa de procedimientos, tales como las unidades de terapia intensiva pediátrica o neonatal.

Es razonable considerar tomar medidas preventivas en las personas alérgicas o sensibilizadas al látex que, por las características de su programa formativo, deben estar expuestas. $^{5}$

\section{OBJETIVO}

Describir la situación clínica de residentes de Pediatría con alergia al látex y las estrategias de prevención en la rotación de Terapia Intensiva Pediátrica y Neonatal.

\section{PACIENTES, MÉTODOS Y RESULTADOS}

Se describen los casos clínicos de tres médicas residentes de Pediatría con diagnóstico de alergia al látex (Tabla 1).

Se definió "alergia al látex" a historia clínica compatible de reacción inmediata con hipersensibilidad tipo I acompañada de inmunoglobulina $\mathrm{E}$ ( $\operatorname{IgE}$ ) específica positiva mediante prueba epicutánea de lectura inmediata y/o sérica. ${ }^{6}$

La prueba epicutánea se realizó de acuerdo con los lineamientos internacionales. ${ }^{7}$ Se utilizó un extracto de látex natural (Allergopharma ${ }^{\circledR}$ ) y la puntura se realizó con lancetas metálicas Diater ${ }^{\circledR}$. Se consideró positivo un tamaño de pápula mayor de $3 \mathrm{~mm}$ superior al control negativo. Las concentraciones séricas de IgE específica para látex fueron obtenidas de muestras de suero. Se realizó mediante ensayo por inmunoabsorción ligado a enzimas (enzymelinked immunosorbent assay; ELISA, por sus siglas en inglés), método Capture Assay Radim Liquid Allergens (CARLA).

Se realizaron 5 reuniones para la discusión, revisión de la bibliografía y el diseño de las estrategias de prevención entre el responsable del programa de residencia, 2 jefes de residentes, el jefe de la Unidad de Cuidados Intensivos Pediátricos, el responsable del Departamento de 
Posgrado y el jefe de la Sección de Alergia.

Cuando existe alergia al látex en el personal de salud, las estrategias propuestas pueden ser limitación o adecuación de las actividades (incluye la reducción de las proteínas de látex en el ambiente, uso de guantes libres de látex y educación a todo el resto de los profesionales sobre la importancia de un ambiente libre de látex) o cambio del lugar laboral (implica cambiar de lugar de trabajo a uno totalmente libre de látex). ${ }^{8}$

Se realizó una capacitación al personal para definir la situación clínica y entrenar en las estrategias de prevención y se incorporaron guantes de nitrilo para todos los procedimientos en la Unidad de Cuidados Intensivos Pediátricos y Terapia Intensiva Neonatal. Se necesitó realizar una segunda capacitación a cuatro integrantes del equipo de salud que no comprendieron o estaban en desacuerdo con modificar sus conductas en el uso de material con látex.

Las tres residentes confirmaron el diagnóstico de alergia al látex (Tabla 1), se les comunicaron las estrategias diseñadas y se obtuvo su aceptación y consentimiento para realizar las rotaciones. Finalmente, se les efectuó una encuesta para que describieran las percepciones en relación con las estrategias de cuidado implementadas.

Se analizaron las siguientes variables: aspectos positivos y negativos de las estrategias que se implementaron, miedos o temores en el resto del programa y en su desarrollo profesional.

\section{RESULTADOS}

Las tres médicas residentes realizaron los 8 meses de rotación en Terapia Intensiva Neonatal y Pediátrica (4 meses en cada una) sin presentar manifestaciones alérgicas.

Los aspectos positivos percibidos en torno a las estrategias diseñadas fueron los siguientes: "sentirse cuidadas", "mejora en la calidad de vida", "que el resto del personal médico tome compromiso con el problema", "sensación positiva por el comienzo de un largo camino de concientización", "ya se tomaron muchas medidas al respecto".

Se percibieron aspectos negativos: "mala predisposición a las nuevas medidas y resistencia al cambio de algunos compañeros de trabajo", "falta de insumos en cuanto a la disponibilidad del tamaño de los guantes estériles", "no se respetan totalmente las medidas en todos los

TABLA 1. Características clínicas de las médicas residentes con alergia al látex

\begin{tabular}{|c|c|c|c|c|c|c|c|}
\hline Caso & Edad & Antecedentes & $\begin{array}{l}\text { Antecedentes } \\
\text { de alergia }\end{array}$ & $\begin{array}{c}\text { Comienzo } \\
\text { síntomas } \\
\text { en programa }\end{array}$ & $\begin{array}{c}\text { Síntomas } \\
\text { durante el } \\
\text { programa }\end{array}$ & $\begin{array}{l}\text { RAST } \\
\text { (ELISA) }\end{array}$ & $\begin{array}{c}\text { Prueba } \\
\text { epicutánea. } \\
\text { Tamaño de } \\
\text { la pápula }\end{array}$ \\
\hline 1 & 29 & $\begin{array}{l}\text { Adenoamigdalectomía. } \\
\text { Asma }\end{array}$ & $\begin{array}{l}\text { Rinitis alérgica. } \\
\text { Edema de labios } \\
\text { en odontólogo. } \\
\text { Eritema y prurito al } \\
\text { usar preservativos } \\
\end{array}$ & Primer año & $\begin{array}{l}\text { Dermatitis y } \\
\text { eritema en } \\
\text { las manos } \\
\text { al usar } \\
\text { guantes }\end{array}$ & $0,97 \mathrm{UI} / \mathrm{ml}$ & $5 \mathrm{~mm}$ \\
\hline 2 & 30 & Peritonitis & $\begin{array}{l}\text { Rinitis alérgica. } \\
\text { Eritema y prurito al } \\
\text { usar preservativos. } \\
\text { Edema de labios } \\
\text { al inflar globos }\end{array}$ & Primer año & $\begin{array}{l}\text { Dermatitis y } \\
\text { eritema en } \\
\text { las manos } \\
\text { al usar guantes }\end{array}$ & $\begin{array}{l}\text { Menor de } \\
0,36 \mathrm{UI} / \mathrm{ml}\end{array}$ & $4 \mathrm{~mm}$ \\
\hline 3 & 27 & $\begin{array}{l}\text { Adenoamigdalectomía. } \\
\text { Cirugías reconstructivas } \\
\text { por accidente (9). } \\
\text { Asma. } \\
\text { Dermatitis atópica }\end{array}$ & $\begin{array}{l}\text { Rinitis alérgica. } \\
\text { Asma. } \\
\text { Edema de labios } \\
\text { en odontólogo. } \\
\text { Eritema y prurito } \\
\text { al usar preservativos }\end{array}$ & Segundo año & $\begin{array}{l}\text { Dermatitis y } \\
\text { eritema en } \\
\text { las manos } \\
\text { al usar guantes } \\
\text { y exacerbación } \\
\text { de asma }\end{array}$ & $\begin{array}{l}\text { Menor de } \\
0,36 \mathrm{UI} / \mathrm{ml}\end{array}$ & $4 \mathrm{~mm}$ \\
\hline
\end{tabular}

RAST: prueba de radioalergoabsorbencia; ELISA: ensayo por inmunoabsorción ligado a enzimas. 
sectores". Asimismo, los miedos o temores percibidos en el ámbito profesional fueron los siguientes: "exposición accidental en otros sectores del hospital", "síntomas cada vez más graves por la exposición", "falta de conocimiento en el propio Hospital". Por último, se percibieron miedos o temores en el ámbito personal: "aparición de otras alergias", "realizar controles médicos u odontológicos", "exposiciones accidentales en ambientes extrahospitalarios".

\section{DISCUSIÓN}

La alergia al látex es un problema de salud que se encuentra en aumento y las estrategias diseñadas permitieron que las residentes completaran las rotaciones de su programa formativo.

En la Argentina, hay 117 residencias pediátricas acreditadas ${ }^{9}$ y su duración tiene una variación de 3 a 4 años. En todos los programas, se incluyen procedimientos que involucran el contacto con productos con látex. ${ }^{10}$

La anamnesis y el índice de sospecha continúan siendo los elementos diagnósticos más importantes. Ante la sospecha clínica de alergia al látex, se debe derivar al paciente a un especialista en alergia para realizar las pruebas diagnósticas necesarias e identificar categorías de riesgo. ${ }^{11}$

Las pruebas epicutáneas son accesibles, inmediatas y económicas comparadas con las pruebas in vitro, por lo tanto, se consideran el patrón de oro para el diagnóstico de alergia al látex. ${ }^{12}$ Cuando se realizan con látex o extractos de sus productos, ofrecen una sensibilidad y especificidad cercanas al 100\% si se utilizan los extractos adecuados. ${ }^{13}$

En las personas alérgicas al látex, la prevención tiene un papel clave, pero la extensión del uso del látex convierte esa prevención en una labor muy compleja. Una de las poblaciones de mayor riesgo son los trabajadores de la salud; alrededor del $3 \%$ al $17 \%$ se encuentra sensibilizado ${ }^{14,15}$ según estudios internacionales.

Aquellos que presentan alergia al látex pueden verse obligados a abandonar sus ámbitos de desempeño o, como en los casos analizados, a implementar medidas dentro de la unidad de trabajo para lograr un ambiente seguro para ellos. Como medida de prevención primaria, se ha probado que la implementación del uso de guantes sin polvo o con bajo contenido de proteínas de látex reduce la sensibilización, el número de síntomas y los niveles de $\operatorname{IgE}$ específica. ${ }^{15}$

Si bien existen estudios publicados que avalan la eficacia de los protocolos de prevención secundaria en pacientes de alto riesgo con alergia al látex, ${ }^{11}$ no hay, hasta el momento, medidas diseñadas dentro de un programa de residencia con médicos alérgicos al látex. Este estudio se propone como una alternativa para reflexionar ante esta problemática.

Las estrategias planteadas procuraron generar un ambiente con bajo contenido de látex que fue suficiente para lograr el objetivo.

Debido al aumento del número de trabajadores de la salud y pacientes con alergia al látex, es importante generar ambientes seguros o con bajo contenido de látex en los hospitales.

Todavía no existen, en la Argentina, hospitales con ambientes que permitan el desarrollo de programas educativos y garanticen la seguridad a los pacientes con esta afección. En este caso, las residentes manifestaron que se habían sentido apoyadas por el sistema de residencia y muchos de sus compañeros durante las rotaciones, sin embargo, persisten situaciones de resistencia y temores en relación con su desarrollo futuro.

El compromiso de todos los responsables involucrados en el proceso educativo fue clave para poder diseñar las estrategias de prevención. Este es un problema de salud creciente que tiene implicancias sanitarias y potencialmente legales, por lo que debe tratarse, de modo necesario, como un problema interdisciplinario ${ }^{1}$ que necesitará de nuevos escenarios para el desarrollo de programas educativos inclusivos que garanticen oportunidades de aprendizaje para los profesionales con esta afección.

\section{Agradecimiento}

Agradecemos la colaboración de los Dres. Nicolás Onna y Danila Labonia.

\section{REFERENCIAS}

1. SternG. Überempfindlichkeit gegen kautschuk als ursache von urticaria und quinckeschem odem. Klin Wochenschr 1927;6:1096-7.

2. Hamilton RG, Adkinson NFJr. Diagnosis of natural rubber latex allergy: multicenter latex skin testing efficacy study. Multicenter Latex Skin Testing Study Task Force. J Allergy Clin Immunol 1998;102(3):482-90.

3. Anda M, Gómez B, Lasa E, et al. Alergia al látex. Manifestaciones clínicas en la población general y reactividad cruzada con alimentos. An Sist Sanit Navar 2003;26(Suppl 2):75-80. 
4. Malamud I. Notas para la evaluación del sistema de residencias médicas. Rev Hosp Ital BAires 2008;28(2):71-4.

5. Merget R, van Kampen V, Sucker K, et al. The German experience 10 years after the latex allergy epidemic: need for further preventive measures in healthcare employees with latexallergy. Int Arch Occup Environ Health 2010;83(8): 895-903.

6. CabañesN, Igea JM, dela Hoz B, et al. Latex Allergy:Position Paper. J Investig Allergol Clin Immunol 2012;22(5):313-30.

7. Bózzola CM. Pruebas cutáneas de lectura inmediata. Técnica, lectura e interpretación. Arch Alerg Inmunol Clin 2002;33(Supl 2):S51-7.

8. Monduzzi G, Franco G. Practising evidence-based occupational health in individual workers: how to deal with a latex allergy problem in a health care setting. Occup Med (Lond) 2005;55(1):3-6.

9. Sociedad Argentina de Pediatría. Consejo de Acreditación de Espacios de Formación CAEF. [Acceso: 30 de junio de 2017]. Disponible en: http:/ / www.sap.org.ar/ docs / caef/
ListadoResidenciasAcreditadas2016.pdf.

10. DuréI. Residencias del equipo de salud. Documento marco 2011. Ministerio de Salud [Acceso: 30 de junio de 2017]. Disponible en: http://www.msal.gov.ar/residencias/ images/stories / descargas / queEs/Documento_Marco_ Residencias_OK.pdf.

11. Parisi CA, B Biló, Bonifazzi F, et al. Alergia al latex. Arch Argent Pediatr 2006;104(6):520-9.

12. Buss ZS, KupekE, Fröde TS. Screening for latex sensitization byquestionnaire: diagnostic performance in health care workers. J Investig Allergol Clin Immunol 2008;18(1):12-6.

13. Turjanmaa K, Reunala T, Räsänen L. Comparison of diagnostic methods in latex surgical glove contact urticaria. Contact dermatitis 1988;19(4):241-7.

14. Sussman GL, Lem D, Liss G, et al. Latex allergy inhousekeeping personal. Ann Allergy Asthma Immunol 1995;74(5):415-8.

15. Yassin MS, Lierl MB, Fischer TJ, et al. Latex allergy in hospital employees. Ann Allergy 1994;72(3):245-9.

"El arte de la Medicina consiste en entretener al paciente con medidas inútiles, mientras la naturaleza trata de curar la enfermedad."

Voltaire 